\title{
Molecular epidemiological typing within the European Gonococcal Antimicrobial Resistance Surveillance Programme reveals predominance of a multidrug- resistant clone
}

S A Chisholm (stephanie.chisholm@hpa.org.uk) ${ }^{1}$, M Unemo $^{2}$, N Quaye ${ }^{1}$, E Johansson ${ }^{2}$, M J Cole ${ }^{1}$, C A Ison ${ }^{1}$, M JW Van de Laar $^{3}$

1. Sexually Transmitted Bacteria Reference Laboratory, Health Protection Agency, Colindale, London, United Kingdom

2. National Reference Laboratory for Pathogenic Neisseria, Department of Laboratory Medicine, Clinical Microbiology, Örebro University Hospital, Örebro, Sweden

3. European Centre for Disease Prevention and Control, Stockholm, Sweden

Citation style for this article:

Chisholm SA, Unemo M, Quaye N, Johansson E, Cole MJ, Ison CA, Van de Laar MJ. Molecular epidemiological typing within the European Gonococcal Antimicrobial Resistance Surveillance Programme reveals predominance of a multidrug-resistant clone. Euro Surveill. 2013;18(3):pii=20358. Available online: http://www. eurosurveillance.org/ViewArticle.aspx?Articleld $=20358$

Article submited on 31 August 2012 / published on 17 January 2013

Treatment of gonorrhoea is threatened by antimicrobial resistance, and decreased susceptibility and resistance to recommended therapies is emerging in Europe. Current associations between resistance and molecular type remain poorly understood. Gonococcal isolates $(n=1,066)$ collected for the 2009 and 2010 European Gonococcal Antimicrobial Surveillance Programme were typed by Neisseria gonorrhoeae multi-antigen sequence typing (NG-MAST). A total of 406 sequence types (STs) were identified, 125 of which occurred in $\geq$ two isolates. Seven major genogroups of closely related STs (varying by $\leq 1 \%$ at just one of the two target loci) were defined. Genogroup 1407 (G1407), observed in 20/21 countries and predominant in $13 / 21$ countries, accounted for $23 \%$ of all isolates and was associated with decreased susceptibility to cefixime and resistance to ciprofloxacin and raised minimum inhibitory concentrations for ceftriaxone and azithromycin. Genogroup 225 (G225), associated with ciprofloxacin resistance, was observed in $10 \%$ of isolates from 19/21 countries. None of the other genogroups were associated with antimicrobial resistance. The predominance of a multidrug-resistant clone (G1407) in Europe is worrying given the recent reports of recommended third generation cephalosporins failing to treat infections with this clone. Identifying associations between ST and antimicrobial resistance aids the understanding of the dissemination of resistant clones within a population and could facilitate development of targeted intervention strategies.

\section{Introduction}

Gonorrhoea continues to present a public health problem within the European Union/European Economic Area (EU/EEA). In 2010, a total of 32,028 cases of gonorrhoea were reported by 28 European countries, giving a rate of 10.4 per 100,000 population [1]. Given the serious implications of gonococcal infection, which include pelvic inflammatory disease, infertility, ectopic pregnancy, early abortion and a fivefold increase in risk of human immunodeficiency virus (HIV) transmission [2], the need for effective treatment and control of gonorrhoea is evident. A significant challenge to this is that during the antibiotic era, the aetiological agent, Neisseria gonorrhoeae, has consistently developed resistance to each antibiotic introduced for widespread empirical treatment [3].

Empirical treatment of gonorrhoea is based on national or regional guidelines, many of which currently recommend third generation cephalosporins as first line therapy. Up until late in 2012, guidelines for Europe recommend $250 \mathrm{mg}$ of ceftriaxone as a single intramuscular dose or $400 \mathrm{mg}$ of cefixime as a single oral dose [4]. These were recently revised to recommend $500 \mathrm{mg}$ ceftriaxone combined with $2 \mathrm{~g}$ azithromycin [5] in response to growing evidence that cephalosporin therapy is becoming compromised by the emergence of isolates exhibiting decreased susceptibility and resistance to the third generation cephalosporins, which have now caused treatment failure in several EU/EEA countries [6-11]. In 2010, the European gonococcal antimicrobial susceptibility programme (Euro-GASP) demonstrated that $9 \%$ of isolates from 21 European countries showed decreased susceptibility to cefixime as well as an increasing trend in minimum inhibitory concentrations (MICs) for ceftriaxone, and a high prevalence of resistance to ciprofloxacin (53\%) and azithromycin (7\%) [12]. In the absence of any alternative antimicrobial treatments, there is clearly an urgent need to develop strategies to limit dissemination of resistant strains to maintain effective treatment.

Molecular epidemiological surveillance has the capacity to provide novel information on the emergence and dissemination of antimicrobial resistant gonococcal 
clones which might facilitate appropriate patient management and targeted intervention strategies. Neisseria gonorrhoeae multi-antigen sequence typing (NG-MAST) is highly discriminatory, reproducible, objective and transferrable and is well suited to applications aiming to answer short term epidemiological questions. Several studies have applied NG-MAST to characterise gonococci exhibiting decreased susceptibility and resistance to various antimicrobials including the third generation cephalosporins [13-21]. However molecular epidemiological surveillance of consecutive isolates of all antimicrobial phenotypes within a population is necessary to explore associations between genotype and antimicrobial resistance profile and to fully understand how resistant strains emerge and disseminate. While several such surveys have been conducted within individual countries in the EU/EEA [22-25] and elsewhere [26-28], most examined gonococcal populations had been collected in 2008 or earlier and, accordingly, associations between sequence type (ST) and antimicrobial susceptibility may no longer be valid. Furthermore examples of decreased susceptibility to recommended third generation cephalosporins were in most cases not circulating in the gonococcal population examined at the time of sampling.

The current study aimed to assess the public health benefit of NG-MAST molecular epidemiological typing of gonococcal isolates as part of Euro-GASP.

\section{Methods}

\section{Isolate collection}

N. gonorrhoeae isolates were selected from the most recent sampling period (2010) within Euro-GASP. The sampling strategy for this multi-centre sentinel surveillance scheme has been described previously [12]. Briefly, countries are requested to submit 55 consecutive gonococcal isolates twice annually (in May/June and November/December) aiming as far as possible to provide isolates representative of the national distribution of cases of gonorrhoea. Where available, countries submitted information on date specimen obtained, specimen site, sex of patient, age, sexual orientation, previously diagnosed with gonorrhoea, and concurrent sexually transmitted infection (STI) diagnosed in this episode.

A total of 1,066 isolates were selected for molecular epidemiological typing from 21 EU/EEA countries. The criteria for selection were that isolates were consecutive to ensure no bias in antimicrobial susceptibility, and that a full antimicrobial resistance profile had been determined as part of EURo-GASP.

Most $(n=828 ; 78 \%)$ were solely from the first sampling period in May/June 2010 in Austria, Belgium, Denmark, France, Germany, Italy, the Netherlands, Sweden and the United Kingdom (UK). Isolates were selected from this sampling period for all other participating countries but further isolates $(n=139 ; 13 \%)$ were additionally selected from the second sampling period (November/ December 2010) for countries submitting low numbers ( $<50$ isolates in the first collection period): Cyprus, Greece, Hungary, Ireland, Romania and Slovakia. The remaining 100 isolates ( $9 \%$ ) were additionally selected from 2009 for countries submitting very low numbers ( $<50$ isolates from both sampling periods in 2010) (Latvia, Malta, Slovenia) or for countries where isolates from the second sampling period in $\mathbf{2 0 1 0}$ were unavailable and ${ }_{50}$ isolates had been submitted in the first sampling period of 2010 (Portugal, Norway and Spain). As Cyprus, Hungary and Romania did not participate in EURO-GASP 2009, only very low numbers (s17) were available from 2010 for inclusion in the current study.

\section{Antimicrobial susceptibility testing}

Gonococcal isolates from each country were submitted on Microbank beads (Pro-Lab Diagnostics, Cheshire, UK) frozen on dry ice to one of three centres (the Health Protection Agency, UK, Örebro University Hospital, Sweden and the Statens Serum Institut, Denmark) for centralised susceptibility testing. All isolates were tested for susceptibility to ciprofloxacin, azithromycin and spectinomycin by a previously decribed breakpoint method [12]. MICs were determined by agar dilution for gentamicin as described $[12,29]$ and by Etest for cefixime and ceftriaxone (AB Biomerieux, Solna, Sweden). The MIC was confirmed by Etest for any isolates resistant to azithromycin by the breakpoint method or showing gentamicin MICs $8 \mathrm{mg} / \mathrm{L}$. All isolates were also tested for penicillinase production using the chromogenic reagent Nitrocefin (Oxoid, Basingstoke, UK).

\section{Determination and analysis of Neisseria gonorrhoeae multi-antigen sequence types} Permission to type isolates submitted for susceptibility testing was obtained from all participating laboratories. All typing was performed at either the Health Protection Agency, UK, or at Örebro University Hospital, Sweden. Isolates originally referred to the Statens Serum Institute in Denmark for susceptibility testing were sent frozen on Microbank beads to the Health Protection Agency, London, UK for typing. A total of 1,066 isolates were typed as described [30] by NG-MAST, which differentiates strains on the basis of sequence variation in fragments of two hypervariable genes, the porin PorB (porB) gene and subunit B of the transferrin binding protein $(t b p B)$ gene. Allele numbers and STs, including any new alleles or STs were assigned via the online NG-MAST database (www.ng-mast.net).

The alleles defining the most frequently observed STs (represented by 10 isolates or more), were compared against all alleles in the dataset to determine if predominant STs were part of a wider collection of closely related types. Similarity of alleles was evaluated in BioNumerics v6.1 by neighbour joining multiple alignment, followed by individual pairwise alignment against the most frequent allele to determine number of base pair (bp) differences. For example, for ST1407 (porB 908, tbpB 110), all porB alleles from isolates 
TABLE 1

Most frequently observed Neisseria gonorrhoeae multi-antigen sequence types in each country, and frequency of the three most common among all isolates $(n=1,066)$, EU/EEA countries, 2009-2010

\begin{tabular}{|c|c|c|c|c|c|c|}
\hline Country & Most frequent ST & STs $n$ & ST1407 n (\%) & ST2992 n (\%) & ST225 n (\%) & Isolates typed n \\
\hline Austria & $\mathrm{ST} 1407$ & 20 & $16(32)$ & $2(4)$ & $\mathrm{o}(0)$ & 50 \\
\hline Belgium & ST1407 & 31 & $7(14)$ & $4(8)$ & $1(2)$ & 50 \\
\hline Cyprus & $\mathrm{ST}_{3128}$ & 5 & $\mathrm{o}(\mathrm{o})$ & $\mathrm{o}(\mathrm{o})$ & $\mathrm{o}(\mathrm{o})$ & 12 \\
\hline Denmark & $\mathrm{ST} 225 / 3158$ & 36 & $3(6)$ & $1(2)$ & $6(12)$ & 50 \\
\hline France & $\mathrm{ST} 2 / 2992$ & 27 & $4(8)$ & $7(14)$ & $2(4)$ & 50 \\
\hline Germany & $\mathrm{ST} 25$ & 18 & $14(28)$ & $\mathrm{o}(0)$ & $\mathrm{o}(\mathrm{o})$ & 50 \\
\hline Greece & $\mathrm{ST}_{5405^{\mathrm{a}} / 5505^{\mathrm{a}}}$ & 24 & $6(12)$ & o (o) & $1(2)$ & 50 \\
\hline Hungary & $\mathrm{ST} 5332^{\mathrm{a}}$ & 10 & $2(12)$ & $\mathrm{o}(\mathrm{o})$ & $\mathrm{o}(0)$ & 17 \\
\hline Ireland & ST2992 & 25 & $3(6)$ & $16(32)$ & o (o) & 50 \\
\hline Italy & ST1407 & 25 & $17(34)$ & $5(10)$ & $\mathrm{o}(\mathrm{o})$ & 50 \\
\hline Latvia & $\mathrm{ST} 3227$ & 14 & $\mathrm{o}(\mathrm{o})$ & $\mathrm{o}(0)$ & $2(7)$ & 29 \\
\hline Malta & $\mathrm{ST} 225$ & 16 & $3(6)$ & $1(2)$ & $23(46)$ & 50 \\
\hline Netherlands & ST1407 & 52 & $16(16)$ & $7(7)$ & $3(3)$ & 100 \\
\hline Norway & $\mathrm{ST} 2992$ & 26 & $7(14)$ & $11(22)$ & o (o) & 49 \\
\hline Portugal & ST1407 & 28 & 7 (14) & $4(8)$ & $\mathrm{o}(0)$ & 50 \\
\hline Romania & ST1407/4120 & 7 & $2(22)$ & o (o) & $\mathrm{o}(\mathrm{o})$ & 9 \\
\hline Slovakia & ST437 & 19 & $2(4)$ & $\mathrm{o}(\mathrm{o})$ & $\mathrm{o}(\mathrm{o})$ & 50 \\
\hline Slovenia & ST1407 & 19 & $14(28)$ & $2(4)$ & $8(16)$ & 50 \\
\hline Spain & ST1407 & 43 & $28(28)$ & 9 (9) & o (o) & 100 \\
\hline Sweden & $\mathrm{ST} 225$ & 31 & $1(2)$ & $3(6)$ & $4(8)$ & 50 \\
\hline United Kingdom & ST1407 & 62 & $14(14)$ & 4(4) & $\mathrm{o}(0)$ & 100 \\
\hline Total N (\%) & & & $166(16)$ & $76(7)$ & $50(5)$ & $1,066(100)$ \\
\hline
\end{tabular}

EEA: European Economic Area; EU: European Union; ST: sequence type.

a New ST.

with $\operatorname{tbp} B$ allele 110 were compared for similarity to por $B$ allele 908 , and all tbp $B$ alleles from isolates containing por $B$ allele 908 were compared for similarity to $t b p B$ allele 110 . Different STs were assigned to a 'genogroup', if one identical allele was shared and the other allele showed $\geq 99 \%$ similarity ( $\leq 5$ bp difference for por $B$ and $\leq 4$ bp for $t b p B$ ). Genogroups were named using the letter $G$ followed by the number of the predominant ST within each group. For example $\mathrm{G}_{1407}$ is the genogroup in which ST1407 is the predominant ST.

\section{Statistical analyses}

Potential associations between genogroups and (i) antimicrobial susceptibilities and (ii) patient characteristics (sex, age and sexual orientation) were explored firstly by univariate analysis and then where appropriate by multivariate analysis using STATA v11.2. For this analysis, patients were subdivided into age groups (o-24 years, 25-34 years, 35-44 years or $\geq 45$ years). Most of the patients in the $0-24$ year age group were aged $15-24$ years (99\%; 360/363), while the remaining three patients were aged $0-7$ years.
For the univariate analysis, crude odds ratios (OR) and 95\% confidence intervals $(\mathrm{Cl})$ were calculated where datasets contained sufficient numbers. A Pearson chi-squared test was used to test if these ORs were significantly different from one (i.e. testing the null hypothesis that there was no difference in odds of resistance/decreased susceptibility between the group in question and the specified baseline group). For datasets where a cell equalled zero, this analysis could not be performed. In these cases of small cell numbers, Fisher's exact test was performed.

The multivariate analysis used logistic regression to model the odds of associations between genogroup and resistance controlling for other variables. The $P$ value produced from the Wald test was used to test the null hypothesis that the odds ratios were not significantly different from one. 


\section{Results}

Frequency of Neisseria gonorrhoeae multi-antigen sequence types

Of the 1,066 isolates typed, 406 different STs were identified, representing 313 porB alleles and $113 \mathrm{tbp} B$ alleles. One hundred and twenty five clusters ( $\geq 2$ isolates with the same ST) and 281 STs represented by a single isolate were identified, and 216 new STs defined. The most frequently observed types (represented by $\geq 10$ isolates) were STs $1407(n=166), 2992(n=76), 225$ $(n=50), 25(n=18), 2(n=17), 359(n=16), 387(n=15), 437$ $(n=14), 3227(n=12), 5405(n=10)$ and $5595(n=10)$, with 5405 and 5595 being new STs. Of these, all had unique por $B$ alleles but some STs shared the same $t b p B$ allele (STs 1407 and 5595, STs 225 and 437, STs 2292 and 359 and STs 387 and 3227 shared, respectively, tbpB alleles 110, 4, 29 and 118).

The prevalence of the three most common STs (1407, 2992 and 225) varied between countries (Table 1). ST1407 accounted for $110 \%$ of isolates in $13 / 21$ countries, whereas ST2992 and ST225 were observed in $110 \%$ of isolates in $3 / 21$ countries in both cases (Table 1). ST1407 was the most frequent ST observed in Austria, Belgium, Italy, the Netherlands, Portugal, Romania, Slovenia, Spain and the UK (Table 1).

\section{Definition and frequency of}

Neisseria gonorrhoeae multi-antigen

sequence typing genogroups

Further analysis of the por $B$ and $t b p B$ alleles of the STs observed in $\geq 10$ isolates showed that several other STs within the total collection were highly related to these, sharing one identical allele and differing by $\leq 1 \%$ at the other allele (in most cases, the porB allele). Seven major genogroups, characterised as $\mathrm{G}_{1407}$, G225, G2992, G25, G387, G359 and G2, were defined (Table 2), encompassing $557(52 \%)$ of isolates. The remaining 509 isolates were represented by 313 STs, of which only STs $5405(n=10), 995(n=9), 951(n=8), 4347$ $(n=8), 5505(n=8), 292(n=7), 2400(n=7), 5402(n=7)$, $384(n=6), 1034(n=6), 1780(n=6)$ and $5598(n=5)$ were observed in $\geq 5$ isolates. The largest and most diverse genogroups were $\mathrm{G} 1407$, comprising ST1407 and 25 STs differing by $\leq 5 \mathrm{bp}$ in the porB allele and two STs by $1 \mathrm{bp}$ in $t b p B$, and $\mathrm{G} 225$ comprising ST225 and 28 other STs differing by $\leq 5 \mathrm{bp}$ at the porB locus (Table 2).

\section{Distribution of Neisseria gonorrhoeae multi-}

antigen sequence typing genogroups

G1407 was observed in all countries examined, with the exception of Latvia, and was the most frequent type, observed in $15-50 \%$ of isolates from Austria, Belgium, Cyprus, Denmark, England and Wales, Greece, Hungary, Italy, Portugal, Romania, Slovakia, Slovenia, Spain and the Netherlands (Figure). In contrast G1407 was comparatively uncommon $(\leq 8 \%)$ in France, Ireland, Malta and Sweden (Figure). G225 was observed in $19 / 21$ countries and was the most frequently observed type in Malta and Sweden and was most common after

\section{TABLE 2}

Neisseria gonorrhoeae multi-antigen sequence types within the seven major genogroups defined based on sequence similarity at $p o r B$ and $t b p B$ alleles, EU/EEA countries, 2009-2010

\begin{tabular}{|c|c|c|c|}
\hline \multirow[b]{2}{*}{$\begin{array}{l}\text { Genogroup } \\
\mathrm{n}(\%)^{\mathrm{a}}\end{array}$} & \multirow[b]{2}{*}{$\begin{array}{l}\text { Predominant } \\
\text { ST (n) }\end{array}$} & \multicolumn{2}{|c|}{ STs within each genogroup (n) } \\
\hline & & $\begin{array}{l}\geq 99 \% \text { similar to } \\
\text { por } B \text { allele } \\
x^{b}\end{array}$ & $\begin{array}{l}\geq 99 \% \\
\text { similar } \\
\text { to } t b p B \\
\text { allele } x^{c}\end{array}$ \\
\hline $\begin{array}{l}\mathrm{G} 1407 \\
248(23)\end{array}$ & $1407(166)$ & $\begin{array}{l}5595(10), 3149(9), 3158(8), \\
3128 \text { (7), } 4120(7), 5570(5), \\
5600(4), 2212(3), 5594(3), \\
3431(1), 3779(1), 4275(1), \\
4359(1), 4951(1), 5588(1), \\
5619(1), 5622(1), 4974(1), \\
5480(1), 5625(1), 5581(1), \\
5649 \text { (1), } 5205(1), 5599(1), \\
5591 \text { (1) }\end{array}$ & $\begin{array}{l}5332(6), \\
4741(4)\end{array}$ \\
\hline $\begin{array}{l}\mathrm{G} 225 \\
107(10)\end{array}$ & $225(50)$ & $\begin{array}{l}437(14), 5463(6), 1132(5), \\
5017(2), 5423(2), 2616(2), \\
3150(2), 5180(2), 205(1), \\
346(1), 1342(1), 1365(1), \\
1399(1), 2687(1), 289(1), \\
1340(1), 2202(1), 3141(1), \\
3153(1), 3952(1), 4315(1), \\
5703(1), 2625(1), 5655(1), \\
3056(1), 4473(1), 5374(1), \\
5330(1)\end{array}$ & \\
\hline $\begin{array}{l}\text { G2992 } \\
84(8)\end{array}$ & $2992(76)$ & $\begin{array}{l}5515(2), 5049 \text { (1) } 5194 \text { (1) } \\
5237 \text { (1), } 5192 \text { (1) } 5385 \text { (1) }\end{array}$ & $5227(1)$ \\
\hline $\begin{array}{l}\mathrm{G} 25 \\
43(4)\end{array}$ & $25(18)$ & $\begin{array}{l}51(7), 3003(4), 356(3), \\
4589(2), 5384(2), 273(1), \\
881(1), 5424(1), 807(1), \\
5341(1), 4338(1), 5172(1)\end{array}$ & \\
\hline $\begin{array}{l}\mathrm{G} 387 \\
37(3)\end{array}$ & $387(15)$ & $\begin{array}{l}3227(12), 5185(4), 5191(1), \\
5486(1), 5503(1), 5186(1), \\
5190(1), 5498 \text { (1) }\end{array}$ & \\
\hline $\begin{array}{l}\mathrm{G} 359 \\
20(2)\end{array}$ & $359(16)$ & 1929 (2), 5485 (1), 1313 (1) & \\
\hline $\begin{array}{l}\mathrm{G} 2 \\
18(2)\end{array}$ & $2(17)$ & $226(1)$ & \\
\hline
\end{tabular}

EEA: European Economic Area; EU: European Union; porB: porin B; $\mathrm{ST}$ : sequence type; $t b p B$ : transferrin binding protein subunit $\mathrm{B}$.

The percentages are calculated from a total of 1,066 isolates.

STs that share an identical $t b p B$ allele with the most frequent ST within the genogroup but vary at the porB allele.

STs that share an identical porB allele with the most frequent ST within the genogroup but vary at the $t b p B$ allele. 


\section{FIGURE}

Proportion of various Neisseria gonorrhoeae multi-antigen sequence typing genogroups within each participating country, EU/EEA countries, 2009-2010

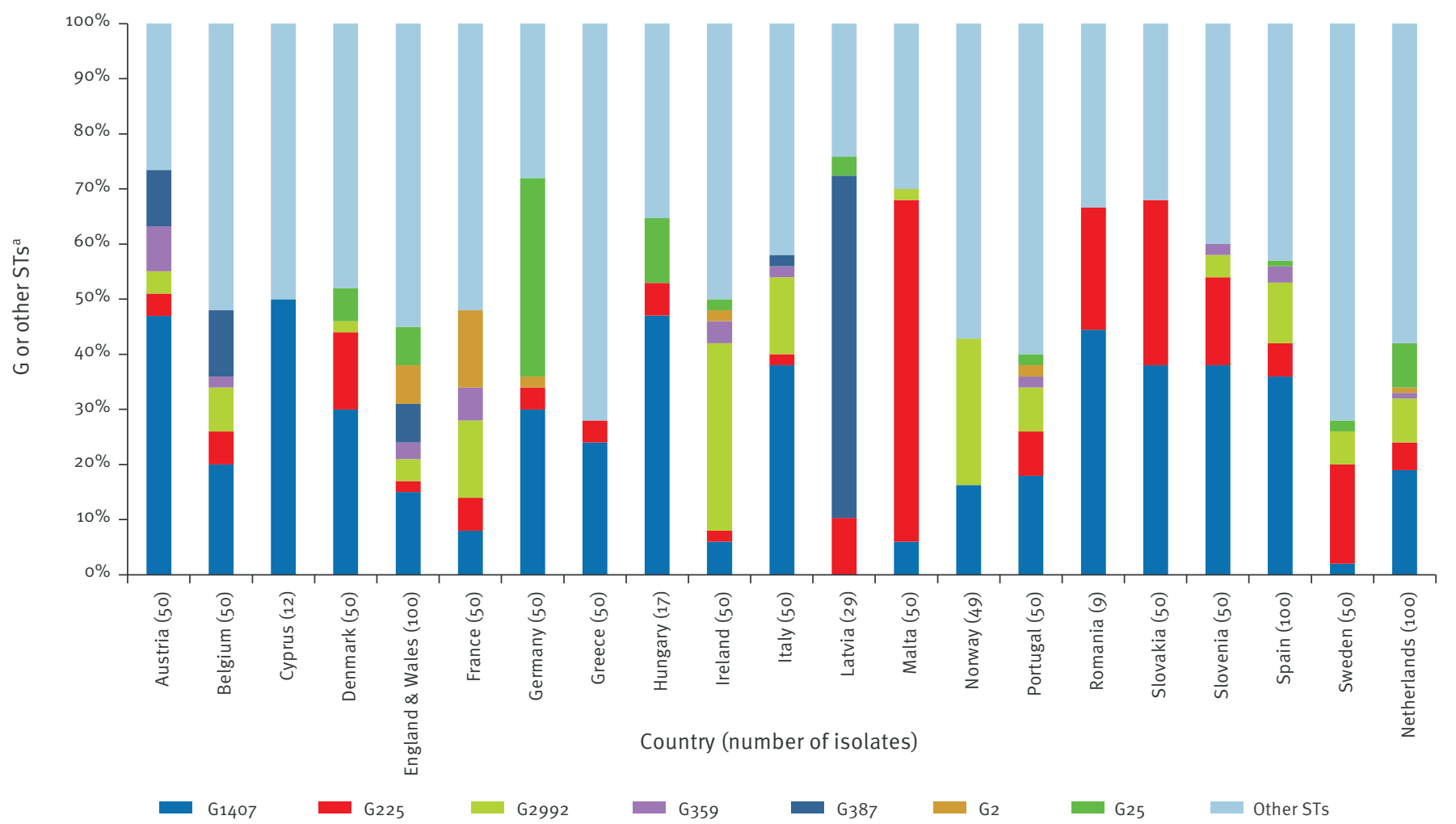

EEA: European Economic Area; EU: European Union; G:genogroup; ST: sequence type.

${ }^{a}$ Other STs refer to the isolates with STs which did not belong to a defined G.

\section{TABLE 3}

Characteristics of patients infected with the most frequently observed Neisseria gonorrhoeae multi-antigen sequence typing genogroups/sequence type, EU/EEA countries, 2009-2010

\begin{tabular}{|c|c|c|c|c|c|c|c|}
\hline Genogroup (n) & $\begin{array}{l}\text { Mean patient } \\
\text { age (range in } \\
\text { years) }\end{array}$ & $\begin{array}{l}\text { Patients of } \\
\text { known sex n }\end{array}$ & $\begin{array}{c}\text { Male patients } \\
\text { n (\%) }\end{array}$ & $\begin{array}{l}\text { Patients of } \\
\text { known sexual } \\
\text { orientation }^{a} n\end{array}$ & $\begin{array}{l}\text { Males of } \\
\text { known sexual } \\
\text { orientation } n\end{array}$ & MSM n (\% $\left.{ }^{b}\right)$ & $\begin{array}{c}\text { Heterosexuals } \\
\text { of both sex } n \\
\left(\% \%^{\mathrm{b}}\right)\end{array}$ \\
\hline $\mathrm{G}_{1407}(248)$ & $33.3(17-69)$ & 244 & $218(89)$ & 155 & 129 & $72(46)$ & $83(54)$ \\
\hline G225 (107) & $29.1(7-56)$ & 104 & $85(82)$ & 78 & 59 & $13(17)$ & $65(83)$ \\
\hline G2992 (84) & $31.7(18-66)$ & 84 & $81(96)$ & 41 & 38 & $36(88)$ & $5(12)$ \\
\hline G25 (43) & $24.6(16-40)$ & 43 & $18(42)$ & 35 & 10 & $2(6)$ & $33(94)$ \\
\hline G387 (37) & $28.6(18-69)$ & 36 & $21(58)$ & 30 & 15 & $0(0)$ & $30(100)$ \\
\hline G359 (20) & $32.9(18-69)$ & 20 & $16(80)$ & 11 & 7 & $5(45)$ & $6(55)$ \\
\hline G2 (18) & $22.1(15-38)$ & 18 & $12(67)$ & 11 & 5 & $\mathrm{o}(0)$ & $11(100)$ \\
\hline $\mathrm{ST}_{5405^{c}(10)}$ & $30.9(6-51)$ & 10 & $8(80)$ & 9 & 7 & $0(0)$ & $9(100)$ \\
\hline All isolates $(1,066)$ & $31.4(0-76)$ & 1,050 & $868(83)$ & 660 & 478 & $222(34)$ & $438(66)$ \\
\hline
\end{tabular}

EEA: European Economic Area; EU: European Union; MSM: men who have sex with men.

a All female patients were included in the heterosexual patient group.

b The percentage is relative to the number of patients of known sexual orientation.

c ST5405 was not found to be part of a larger genogroup. 
G1407 in Denmark, Romania, Slovakia and Slovenia, accounting for $14-30 \%$ of isolates (Figure). G2992 was observed in $14 / 21$ countries and predominated in Ireland and Norway, while G25 predominated in Germany (Figure).

\section{Neisseria gonorrhoeae multi-antigen sequence} typing genogroups and epidemiological

characteristics of linked patients

The most frequently collected variables were age and sex, known for 1,030 and 1,050 patients, respectively. Sexual orientation was known for only 586 patients (478 males and 108 females) (Table 3).

Statistical analysis showed there was no clear association with age category for most genogroups, with the exception of $\mathrm{G}_{1407}$ which was significantly less common in patients aged 25 years (OR: 0.43; p<0.0001, chi-squared test), whereas $\mathrm{G}_{2}$ was significantly more common in this younger group (OR: 15.63; $p=0.0004$, chi-squared test). The proportion of males infected was significantly lower for G25 (OR: 0.13; p<0.0001, chi-squared test) and G387 (OR: 0.27; $p=0.0001$, chisquared test) but was significantly higher for G2992 (OR: 6.14; $p=0.0005$, chi-squared test) and $\mathrm{G}_{1407}$ (OR: 2.01; $p=0.0017$, chi-squared test). Where sexual orientation was known, statistical associations were observed between men who have sex with men (MSM) and infection with G2992 (OR: 15.86; p<0.0001, chi-squared test) and G1407 (OR: 1.75; $p=0.003$, chisquared test), although $54 \%$ of patients infected with G1407 were heterosexual (Table 3). MSM were less frequently infected with G225 (OR: 0.039; $p=0.0015$, chisquared test), G387 (p<0.0001, Fisher's exact test) and $\mathrm{G} 2$ ( $p=0.01$, Fisher's exact test).

Neisseria gonorrhoeae multi-antigen sequence typing genogroups or sequence types and antimicrobial resistance

The consensus antimicrobial susceptibility category or modal MIC was calculated for all genogroups/STs represented by $\geq 10$ isolates (Table 4 ). Most deviation from the consensus was observed for azithromycin, which supplementary testing by Etest showed was due to isolates exhibiting MICs close to the breakpoint for resistance $(1.0 \mathrm{mg} / \mathrm{L})$.

The modal cefixime MIC was raised for $\mathrm{G} 1407$ compared with other genogroups (Table 4 ), with $77 / 248$ isolates (31\%) showing decreased susceptibility to cefixime (MIC >0.125 mg/L) while $176 / 248$ isolates (71\%) had MICs $\geq 0.125 \mathrm{mg} / \mathrm{L}$. Almost all isolates (239/248; 96\%) had cefixime MICs $\geq 0.06 \mathrm{mg} / \mathrm{L}$. The association between G1407 and decreased susceptibility to cefixime was highly significant (OR: 17.9; p<0.0001, chi-squared test) by univariate analysis and by multivariate analysis to control for patient age, sex and sexual orientation (OR: 31.2; p<0.0001, chi-squared test). There were no examples of decreased ceftriaxone susceptibility (MIC >0.125 $\mathrm{mg} / \mathrm{L}$ ), but the modal MIC was raised for $\mathrm{G} 1407 \mathrm{com}$ pared with other genogroups (Table 4). All isolates of
G1407 were ciprofloxacin resistant (p<0.0001, Fisher's exact test). While the consensus azithromycin susceptibility for $\mathrm{G} 1407$ was sensitive, the proportion of isolates showing MICs just above the breakpoint and so in the resistant category was significant after controlling for patients age, sex and sexual orientation (OR: 11.5; p $<0.0001$, chi-squared test). Decreased cefixime susceptibility was observed in a further nine STs representing 10 isolates in the total study population. Of these, seven isolates shared $t b p B$ allele 110 but the six por $B$ alleles differed from allele 908 by $>5$ bp. One isolate possessed por $B$ allele 908 but differed markedly at the $t b p B$ allele from 110 . The STs of the remaining two isolates (STs 3168 and 5606) appeared unrelated to ST1407.

All isolates belonging to $\mathrm{G} 225$ were resistant to ciprofloxacin, indicating a statistically significant association by Fisher's exact test ( $p<0.0001)$. All ten isolates of $\mathrm{ST}_{5405}$ were resistant to ciprofloxacin also (Table 4), significant association by Fisher's exact test $(p=0.0032)$.

\section{Discussion}

To our knowledge this is the first study to supplement a regional antimicrobial resistance surveillance programme with molecular typing to provide enhanced surveillance of gonorrhoea. This approach has not only provided novel information on NG-MAST STs circulating in 21 of the 30 EU/EEA countries, but also provides insight into the dissemination of antimicrobial resistant gonococcal clones within the region.

While considerable diversity of gonococcal STs exists both within and between countries, some STs predominate, and this is even more apparent if STs are grouped into genogroups to facilitate robust statistical analyses. Most notably, G1407 accounted for $23 \%$ of isolates overall, and predominated in 13/21 countries. Relatedness of NG-MAST STs has been considered in comparatively few studies and multiple approaches are described $[14,15,21,25,31,32]$. Failure to consider relatedness of STs could lead to the prevalence of clonal groups being underestimated and reduces the power of any statistical analyses exploring associations between organism and/or patient characteristics and STs. Our approach was to consider strains highly related if strains varied at just one allele by $\leq 1 \%$. Use of such a high cut-off ensures only very closely related strains are clustered, but given the variation in approaches described thus far, there is a need to agree a standard approach in the near future to allow comparability between studies.

Most molecular surveys of consecutive gonococcal isolates from earlier sampling periods ranging between 2003 to 2005 did not demonstrate predominance of ST1407 or related types in individual European countries $[24,25,33,34]$ or elsewhere $[26,28]$. In contrast ST1407 was documented in more recent surveys in Portugal [25] and Norway [23], and a centre that 
Consensus antimicrobial susceptibility results for Neisseria gonorrhoeae multi-antigen sequence typing genogroups/sequence type represented by 10 or more isolates, EU/EEA countries, 2009-2010

\begin{tabular}{|c|c|c|c|c|c|c|c|c|}
\hline \multirow{2}{*}{$\begin{array}{l}\text { Genogroup } \\
\text { or ST }\end{array}$} & \multirow{2}{*}{$\begin{array}{c}\text { Isolates } \\
n\end{array}$} & \multirow{2}{*}{$\begin{array}{l}\text { Beta- } \\
\text { lactamase }\end{array}$} & \multicolumn{3}{|c|}{$\begin{array}{l}\text { Consensus resistance category } \\
\text { (Isolates differing from the consensus) }\end{array}$} & \multicolumn{3}{|c|}{ Modal MIC mg/L (range) } \\
\hline & & & Azithromycin & Ciprofloxacin & Spectinomycin & Gentamicin & Cefixime $^{\mathrm{a}}$ & Ceftriaxone $^{\mathrm{a}}$ \\
\hline $\mathrm{G}_{1407}$ & 248 & Negative & $S\left(44^{b}\right)$ & $\mathrm{R}(0)$ & $\mathrm{S}(0)$ & $8(4-16)$ & $\begin{array}{c}0.125 \\
(0.032-0.25)\end{array}$ & $\begin{array}{c}0.047 \\
(0.008-0.125)\end{array}$ \\
\hline G225 & 107 & Negative & $S\left(1^{c}\right)$ & $\mathrm{R}(\mathrm{o})$ & $\mathrm{S}(\mathrm{o})$ & $8(4-16)$ & $\begin{array}{c}0.023 \\
((0.016-0.064)\end{array}$ & $\begin{array}{c}0.016 \\
(0.003-0.047)\end{array}$ \\
\hline G2992 & 84 & Negative & $S\left(14^{d}\right)$ & $S(1)$ & $S(0)$ & $8(2-8)$ & $\begin{array}{c}0.023 \\
((0.016-0.064)\end{array}$ & $\begin{array}{c}0.006 \\
(0.002-0.016)\end{array}$ \\
\hline G25 & 43 & Negative & $S(0)$ & $S(1)$ & $\mathrm{S}(\mathrm{o})$ & $8(4-8)$ & $\begin{array}{c}<0.016 \\
(<0.016-0.064)\end{array}$ & $\begin{array}{c}0.003 \\
((0.002-0.016)\end{array}$ \\
\hline G387 & 37 & Negative & $S(0)$ & $S(0)$ & $\mathrm{S}(0)$ & $8(4-8)$ & $\begin{array}{c}<0.016 \\
(<0.016-0.016)\end{array}$ & $\begin{array}{c}<0.002 \\
(<0.002-0.002)\end{array}$ \\
\hline G359 & 20 & Negative & $S\left(1^{c}\right)$ & $S(0)$ & $S(0)$ & $8(4-16)$ & $\begin{array}{c}0.064 \\
(<0.016-0064)\end{array}$ & $\begin{array}{c}0.008 / 0.012 \\
(0.004-0.023)\end{array}$ \\
\hline $\mathrm{G}_{2}$ & 18 & Negative & $\mathrm{S}(\mathrm{o})$ & $S(0)$ & $S(0)$ & $4(2-8)$ & $\begin{array}{c}<0.016 \\
(<0.016-0.032) \\
\end{array}$ & $\begin{array}{c}0.003 \\
((0.002-0.012)\end{array}$ \\
\hline ST5405 & 10 & Negative & $S(0)$ & $R(0)$ & $S(0)$ & $8(4-8)$ & $\begin{array}{c}<0.016 \\
(<0.016-0.023)\end{array}$ & $\begin{array}{c}0.004 / 0.006 \\
(0.003-0.006)\end{array}$ \\
\hline
\end{tabular}

EEA: European Economic Area; EU: European Union; MIC: minimum inhibitory concentration; S: susceptible; ST: sequence type; R: resistant.

Cefixime and Ceftriaxone MICs are described according to the Etest scale.

40 of the 44 with an R category were within one doubling dilution of the breakpoint.

Isolates that differ from the consensus were within one doubling dilution of the breakpoint.

10 of the 14 with an R category were within one doubling dilution of the breakpoint.

performs molecular typing of all cases of gonorrhoea in Scotland showed that ST1407 first emerged in 2007 and by 2009 accounted for $15.4 \%$ of cases [35]. The current study provides further evidence that the wide dissemination of $\mathrm{ST}_{1407}$ in the EU is likely to be a recent phenomenon. ST1407 and related STs are known to be distributed globally, documented in studies specifically investigating decreased susceptibility and resistance to third generation cephalosporins, in Europe $[6,9,13,14,19]$, the United States [20], Canada [21], Australia [18], Hong Kong [17] and Taiwan [36] and in a survey conducted in Japan [27]. ST1407 has also caused most of the treatment failures with third generation cephalosporins in EU/EEA countries [6-11].

Our study demonstrates that there appear to be no isolates of $\mathrm{G} 1407$ that are highly sensitive to cefixime, with $96 \%$ of isolates showing MICs $\geq 0.06 \mathrm{mg} / \mathrm{L}$, and presents the first clear evidence that all $\mathrm{G}_{1407}$ isolates circulating currently in the general gonococcal population in the EU/EEA are ciprofloxacin resistant, strongly associated with decreased susceptibility/ resistance to cefixime and show raised MICs for ceftriaxone and azithromycin. This highlights the potential of molecular epidemiological typing as a tool to predict antimicrobial resistance, as it is evident that ciprofloxacin and cefixime in particular would be inappropriate regimes to treat $\mathrm{G}_{1407}$ infection. Two isolates were identified that exhibited decreased susceptibility to cefixime but were considered unrelated to G1407. The low incidence of these types may suggest they represent less biologically fit gonococcal clones and/or have recently been imported from regions such as the Far East, where greater diversity in STs showing cefixime decreased susceptibility is reported [15-17,36]. ST1407 is known to be part of a major globally predominant clone, multilocus sequence type (MLST) ST1901 which probably originated in Japan [11], and recent analysis of ten additional genomic markers has provided further evidence of the clonal nature of ST1407 and closely related NG-MAST STs [32]. MLST ST7363 is also reported as a major clone associated with decreased susceptibility and resistance to cephalosporins, which also has shown its ability to develop high-level resistance to cefixime and ceftriaxone [37]. MLST was not performed in the current study but is an excellent means of determining longer-term evolutionary relationships between strains [38] and so could help to resolve the lineage of these non-G1407 isolates. 
Treatment failure in patients infected with gonococci exhibiting cefixime MICs of the levels in the current study have been documented in the UK [10], Norway [9] and Austria [6] and all belonged to G1407. One ST1407 isolate with high-level resistance to cefixime $(\mathrm{MIC}=4$ $\mathrm{mg} / \mathrm{L}$ ) and ceftriaxone $(\mathrm{MIC}=1-2 \mathrm{mg} / \mathrm{L})$, also associated with treatment failure using cefixime, has been described from France [11]. This strain demonstrates that the $\mathrm{ST} 1407$ clone can by a single additional mutation in its penicillin-binding protein 2 (penA) mosaic gene develop high-level resistance to both cefixime and ceftriaxone, which is the last remaining option for single antimicrobial empiric treatment of gonorrhoea [11]. The observed predominance of ST1407 and related STs in many EU/EEA countries is therefore worrying given their potential to be therapeutically challenging. While shown to be associated with MSM, it is evident that $\mathrm{G}_{1407}$ is circulating in the heterosexual population also and, accordingly, the risk of future treatment failure is not restricted to any one patient group. $\mathrm{G} 1407$ therefore represents a potential major public health problem if it continues to disseminate without any measures taken to restrict this.

G225 was the most prevalent genogroup after G1407 in the current study. ST225 has been reported as a highly prevalent strain in EU/EEA countries [22,25,33,34], with a confirmed association with ciprofloxacin resistance $[22,25,33]$. Given that most studies began sampling as early as 2003 to 2005 , the continued persistence of G225 by 2010 indicates that it is a highly successful, stable NG-MAST clone. Interestingly, a recent Canadian study of isolates showing decreased susceptibility to cefixime and/or ceftriaxone showed that $19 \%$ of isolates were ST225 [21]. All isolates of G225 in the current study were sensitive to cefixime and ceftriaxone, but the potential for this strain to show decreased susceptibility is of concern given its potential to predominate and persist. While strong associations between ST225 and MSM are reported [25,34], these were not confirmed in all studies [39] including this one. This could indicate a shift in the distribution of G225 since the earlier typing studies, with possible bridging between homosexual and heterosexual networks. It is acknowledged however that the completeness of the sexual orientation variable in the current study was low, which may have introduced a bias into this analysis.

None of the other major genogroups defined in the current study showed an association with resistance to the antimicrobials tested, but G2, G25, G359 and G387 would appear to be particularly persistent strains also, all having been reported in a molecular survey in 2004 [34]. Individual STs from these groups have been reported in other studies which confirmed that these were susceptible to the antimicrobials tested $[22,25,33]$. As observed previously [34], genogroups $\mathrm{G}_{2}, \mathrm{G}_{2} 5$ and $\mathrm{G}_{3} 87$ were associated with heterosexual patients, demonstrating their persistence within this patient population. In contrast other genogroups (G2992, G1407) were shown to be associated with
MSM. While ST1407 has been reported in a high proportion of MSM previously [13], to our knowledge this is the first time ST2992 and related types has been reported as a potentially MSM associated type. None of the molecular epidemiological surveys conducted to date have reported ST2992 as a prevalent ST [24$26,28,33,34]$. G2992 accounted for $8 \%$ of all isolates in our study and while widely distributed, it was particularly predominant in Ireland and Norway, which may indicate an outbreak within MSM, although further longitudinal surveillance of a sufficiently representative sample would be required to verify this.

In our study the selection criteria for isolates were based on accepting from each country consecutive isolates with a full antimicrobial profile. There were no exclusion criteria based on epidemiological characteristics and all patient ages were included. One of the age groups used for the analyses spanned o to 24 year-olds however only three of the 363 patients in this group were less than 15 years-old. The inclusion of isolates from the latter patients was valid for the antimicrobial resistance analyses. Only one of the three patients was related to a common genogroup ( $G_{225}$ ) therefore including these three patients should have had little effect on the overall statistical analyses.

In conclusion, this study shows clear associations between antimicrobial resistance and molecular type. The use of molecular epidemiological typing to predict antimicrobial resistance and follow the spread of antimicrobial resistant gonococcal clones has added public health benefits as it can aid understanding of the dissemination of resistance within a population and facilitate development of targeted intervention strategies, for example by ensuring patients infected with strains likely to be therapeutically challenging are managed with aggressive therapy and test of cure. Additionally if the prediction of antimicrobial resistance was sufficiently reliable this approach could directly impact on appropriate management of patients for whom culture and associated antimicrobial susceptibility testing has not been performed. While it is acknowledged that sample representativeness is a limitation of the current study [12], additional molecular epidemiological typing has successfully demonstrated a 'proof of principle' that this approach could be valid and have real public health value. However for this to be effective, ongoing, frequent molecular typing surveillance will be essential, as there is continual potential for novel STs and novel antimicrobial susceptibility profiles to emerge. Future work should focus on longitudinal typing of a representative sample to monitor stability of associations between ST and antimicrobial resistance and/or epidemiological characteristics, and to identify temporal changes and emergence of novel STs. 
Funding

This work was supported by the European Centre for Disease Prevention and Control (call for tender number 0J/2009/03/11-PROC/2009/006), which coordinates the European STI Expert Network, including the STI microbiology programme.

\section{Acknowledgements}

We are indebted to all national nominated contact points within the European STI Expert Network and to their colleagues for their continued support and provision of gonococcal isolates as part of the European Gonococcal Antimicrobia Surveillance Programme (Euro-GASP). We thank all members of the European Expert Group on Molecular Typing for their contribution to the original strategy for European typing. We are grateful to Steen Hoffman, Mrs Lene Berthelsen and Miss Ronza Hadad for their invaluable contribution to the antimicrobial susceptibility testing, and to Miss Katy Town for her valued assistance with the statistical analyses.

\section{References}

1. European Centre for Disease Prevention and Control (ECDC). Sexually Transmitted Infections in Europe, 1990-2010. Stockholm: ECDC; 2012. Available from: http://www.ecdc. europa.eu/en/publications/Publications/201206-SexuallyTransmitted-Infections-Europe-2010.pdf

2. World Health Organization (WHO). Emergence of multi-drug resistant Neisseria gonorrhoeae - Threat of global rise in untreatable sexually transmitted infections. WHO/RHR/11.14 2011. Fact sheet. Geneva:WHO; 2011. Available from: http:// www.who.int/reproductivehealth/publications/rtis/who_ rhr_11_14/en/index.html

3. Lewis DA. The Gonococcus fights back: is this time a knock out? Sex Transm Infect. 2010;86(6):415-21.

4. Bignell CJ; IUSTI/WHO. 2009 European (IUSTI/WHO) guideline on the diagnosis and treatment of gonorrhoea in adults. Int.J.STD AIDS. 2009;20(7), 453-7.

5. Bignell C, Unemo M. 2012 European Guideline on the Diagnosis and Treatment of Gonorrhoea in Adults. The International Union against Sexually Transmitted Infections (IUSTI); 2012:124. Available from: http://www.iusti.org/regions/Europe/ pdf/2012/Gonorrhoea_2012.pdf

6. Unemo M, Golparian D, Stary A, Eigentler A. First Neisseria gonorrhoeae strain with resistance to cefixime causing gonorrhoea treatment failure in Austria, 2011. Euro Surveill. 2011;16(43): pii=19998. Available from: http://www. eurosurveillance.org/ViewArticle.aspx?Articleld=19998

7. Unemo M, Golparian D, Potočnik M, Jeverica S. Treatment failure of pharyngeal gonorrhoea with internationally recommended first-line ceftriaxone verified in Slovenia, September 2011. Euro Surveill. 2012;17(25): $\mathrm{pii}=20200$. Available from: http://www.eurosurveillance.org/ViewArticle. aspx?Articleld $=20200$

8. Unemo M, Golparian D, Hestner A. Ceftriaxone treatment failure of pharyngeal gonorrhoea verified by international recommendations, Sweden, July 2010. Euro Surveill. 2011;16(6):pii=19792. Available from: http://www. eurosurveillance.org/ViewArticle.aspx?Articleld=19792

9. Unemo M, Golparian D, Syversen G, Vestrheim DF, Moi H. Two cases of verified clinical failures using internationally recommended first-line cefixime for gonorrhoea treatment, Norway, 2010. Euro Surveill. 2010;15(47):pii=19721. Available from: http://www.eurosurveillance.org/ViewArticle. aspx?Articleld $=19721$

10. Ison CA, Hussey J, Sankar KN, Evans J, Alexander S. Gonorrhoea treatment failures to cefixime and azithromycin in England, 2010. Euro Surveill. 2011;16(14): $\mathrm{pii}=19833$. Available from: http://www.eurosurveillance.org/ViewArticle. aspx?Articleld=19833

11. Unemo M, Golparian D, Nicholas R, Ohnishi M, Gallay A, Sednaoui P. High-level cefixime- and ceftriaxone-resistant Neisseria gonorrhoeae in France: novel penA mosaic allele in a successful international clone causes treatment failure. Antimicrob Agents Chemother. 2012;56(3):1273-1280.

12. European Centre for Disease Prevention and Control (ECDC). Gonococcal antimicrobial susceptibility surveillance in Europe,
2010. Stockholm:ECDC; 2012. Available from: http://www.ecdc. europa.eu/en/publications/Publications/1206-GonococcalAMR.pdf

13. Chisholm SA, Alexander S, Desouza-Thomas L, MaclureWebster E, Anderson J, Nichols T et al. Emergence of a Neisseria gonorrhoeae clone showing decreased susceptibility to cefixime in England and Wales. J Antimicrob Chemother. 2011;66(11):2509-12.

14. Golparian D, Hellmark B, Fredlund H, Unemo M. Emergence, spread and characteristics of Neisseria gonorrhoeae isolates with in vitro decreased susceptibility and resistance to extended-spectrum cephalosporins in Sweden. Sex Transm Infect. 2010;86(6):454-460.

15. Liao M, Gu WM, Yang Y, Dillon JA. Analysis of mutations in multiple loci of Neisseria gonorrhoeae isolates reveals effects of PIB, PBP2 and MtrR on reduced susceptibility to ceftriaxone. J Antimicrob Chemother. 2011;66(5):1016-23.

16. Lee SG, Lee H, Jeong SH, Yong D, Chung GT, Lee YS et al. Various penA mutations together with mtrR, porB and ponA mutations in Neisseria gonorrhoeae isolates with reduced susceptibility to cefixime or ceftriaxone. J Antimicrob Chemother. 2010;65(4):669-75.

17. Lo JY, Ho KM, Lo AC. Surveillance of gonococcal antimicrobial susceptibility resulting in early detection of emerging resistance. J Antimicrob Chemother. 2012;67(6):1422-6.

18. Tapsall JW, Ray S, Limnios A. Characteristics and population dynamics of mosaic penA allele-containing Neisseria gonorrhoeae isolates collected in Sydney, Australia, in 20072008. Antimicrob Agents Chemother. 2010;54(1):554-6.

19. Carannante A, Prignano G, Cusini M, Matteelli A, Dal Conte I, Ghisetti V et al. Cefixime and ceftriaxone susceptibility of Neisseria gonorrhoeae in Italy from 2006 to 2010. Clin Microbiol Infect. 2012;18(6):558-64.

20. Pandori M, Barry PM, Wu A, Ren A, Whittington WL, Liska $S$ et al. Mosaic penicillin-binding protein 2 in Neisseria gonorrhoeae isolates collected in 2008 in San Francisco, California. Antimicrob Agents Chemother. 2009;53(9):4032-4.

21. Martin I, Sawatzky P, Allen V, Hoang L, Lefebvre B, Mina N et al. Emergence and characterization of Neisseria gonorrhoeae isolates with decreased susceptibilities to ceftriaxone and cefixime in Canada: 2001-2010. Sex Transm Dis. 2012;39(4):316-23.

22. Palmer HM, Young H, Graham C, Dave J. Prediction of antibiotic resistance using Neisseria gonorrhoeae multi-antigen sequence typing. Sex Transm Infect. 2008;84(4):280-4.

23. Hjelmevoll SO, Golparian D, Dedi L, Skutlaberg DH, Haarr E, Christensen A et al. Phenotypic and genotypic properties of Neisseria gonorrhoeae isolates in Norway in 2009: antimicrobial resistance warrants an immediate change in national management guidelines. Eur J Clin Microbiol Infect Dis. 2012;31(6):1181-6.

24. Starnino S, Suligoi B, Regine V, Bilek N, Stefanelli P. Neisseria gonorrhoeae Italian Study Group, et al. Phenotypic and genotypic characterization of Neisseria gonorrhoeae in parts of Italy: detection of a multiresistant cluster circulating in a heterosexual network. Clin Microbiol Infect. 2008;14(10):949-54.

25. Florindo C, Pereira R, Boura M, Nunes B, Paulino A, Gomes JP et al. Genotypes and antimicrobial-resistant phenotypes of Neisseria gonorrhoeae in Portugal (2004-2009). Sex Transm Infect. 2010;86(6):449-53.

26. Unemo M, Vorobieva V, Firsova N, Ababkova T, Leniv I, Haldorsen BC et al. Neisseria gonorrhoeae population in Arkhangelsk, Russia: phenotypic and genotypic heterogeneity. Clin Microbiol Infect. 2007;13(9):873-8.

27. Tanaka M, Koga Y, Nakayama H, Kanayama A, Kobayashi I, Saika T et al. Antibiotic-resistant phenotypes and genotypes of Neisseria gonorrhoeae isolates in Japan: identification of strain clusters with multidrug-resistant phenotypes. Sex Transm Dis. 2011;38(9):871-5.

28. Wong WW, Huang CT, Li LH, Chiang CC, Chen BD, Li SY. Molecular epidemiological identification of Neisseria gonorrhoeae clonal clusters with distinct susceptibility profiles associated with specific groups at high risk of contracting human immunodeficiency virus and syphilis. J Clin Microbiol. 2008;46(12):3931-4.

29. Chisholm SA, Quaye N, Cole MJ, Fredlund H, Hoffmann S, Jensen IS et al. An evaluation of gentamicin susceptibility of Neisseria gonorrhoeae isolates in Europe. J Antimicrob Chemother. 2011;66(3):592-5.

30. Martin IM, Ison CA, Aanensen DM, Fenton KA, Spratt BG. Rapid sequence-based identification of gonococcal transmission clusters in a large metropolitan area. J Infect Dis. 2004;189(8):1497-505. 
31. Buono S, Wu A, Hess DC, Carlson JS, Rauch L, Philip SS et al. Using the Neisseria gonorrhoeae multiantigen sequence-typing method to assess strain diversity and antibiotic resistance in San Francisco, California. Microb Drug Resist. 2012;18(5):510-7.

32. Hess D, Wu A, Golparian D, Esmaili S, Pandori W, Sena E et al. Genome sequencing of a Neisseria gonorrhoeae isolate of a successful international clone with decreased susceptibility and resistance to extended-spectrum cephalosporins.

Antimicrob Agents Chemother. 2012;56(11):5633-41.

33. Olsen B, Hadad R, Fredlund H, Unemo M. The Neisseria gonorrhoeae population in Sweden during 2005-phenotypes, genotypes and antibiotic resistance. APMIS. 2008;116(3):181-9.

34. Risley CL, Ward H, Choudhury B, Bishop CJ, Fenton KA, Spratt BG et al. Geographical and demographic clustering of gonorrhoea in London. Sex Transm Infect. 2007;83(6):481-7.

35. Eastick K. Gonococcal antibiotic surveillance in Scotland (GASS): prevalence, patterns and trends in 2009. Health Protection Scotland Weekly Report. 2010;44(34).

36. Huang CT, Yen MY, Wong WW, Li LH, Lin KY, Liao MH et al. Characteristics and dissemination of mosaic penicillin-binding protein 2-harboring multidrug-resistant Neisseria gonorrhoeae isolates with reduced cephalosporin susceptibility in northern Taiwan. Antimicrob Agents Chemother. 2010;54(11):4893-5.

37. Ohnishi M, Golparian D, Shimuta K, Saika T, Hoshina S, Iwasaku $\mathrm{K}$ et al. Is Neisseria gonorrhoeae initiating a future era of untreatable gonorrhea?: detailed characterization of the first strain with high-level resistance to ceftriaxone. Antimicrob Agents Chemother. 2011;55(7):3538-45.

38. Vidovic S, Horsman GB, Liao M, Dillon JA. Influence of conserved and hypervariable genetic markers on genotyping circulating strains of Neisseria gonorrhoeae. PLoS One. 2011;6(12):e28259.

39. Martin IM, Ison CA, Aanensen DM, Fenton KA, Spratt $B G$. Changing epidemiologic profile of quinoloneresistant Neisseria gonorrhoeae in London. J Infect Dis. 2005;192(7):1191-5. 